
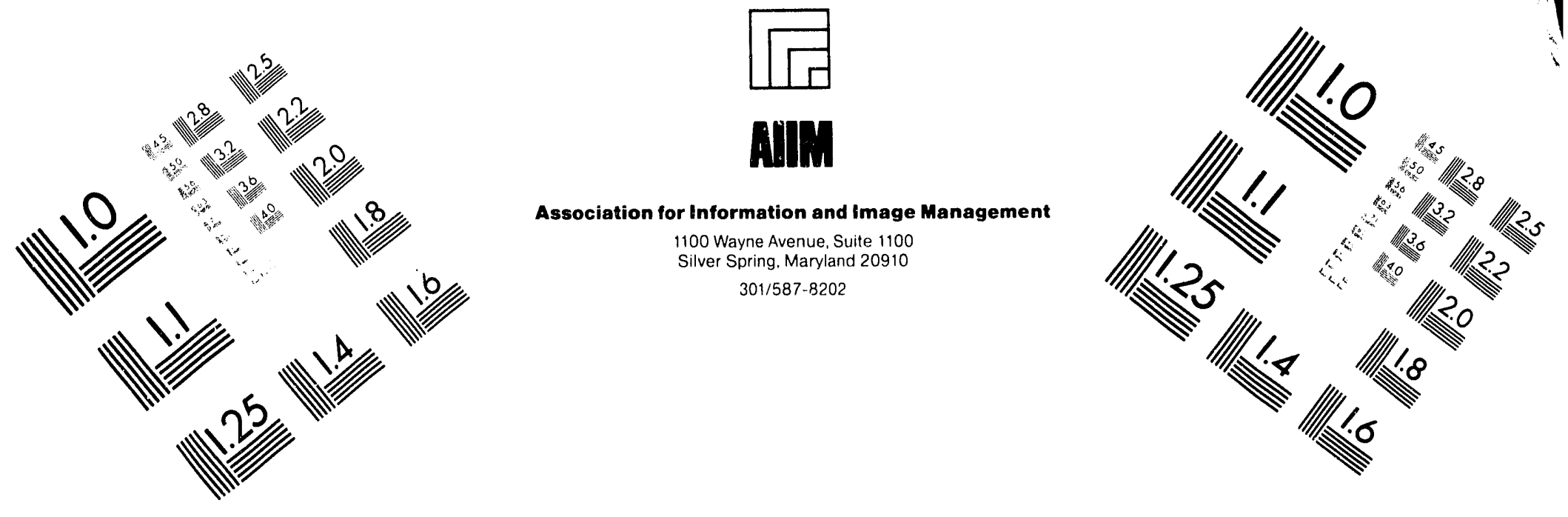

\title{
Centimeter
}

$\begin{array}{llllllllllllllll}1 & 2 & 3 & 4 & 5 & 6 & 7 & 8 & 9 & 10 & 11 & 12 & 13 & 14 & 15 & \mathrm{~mm}\end{array}$ $\mid$ Inches
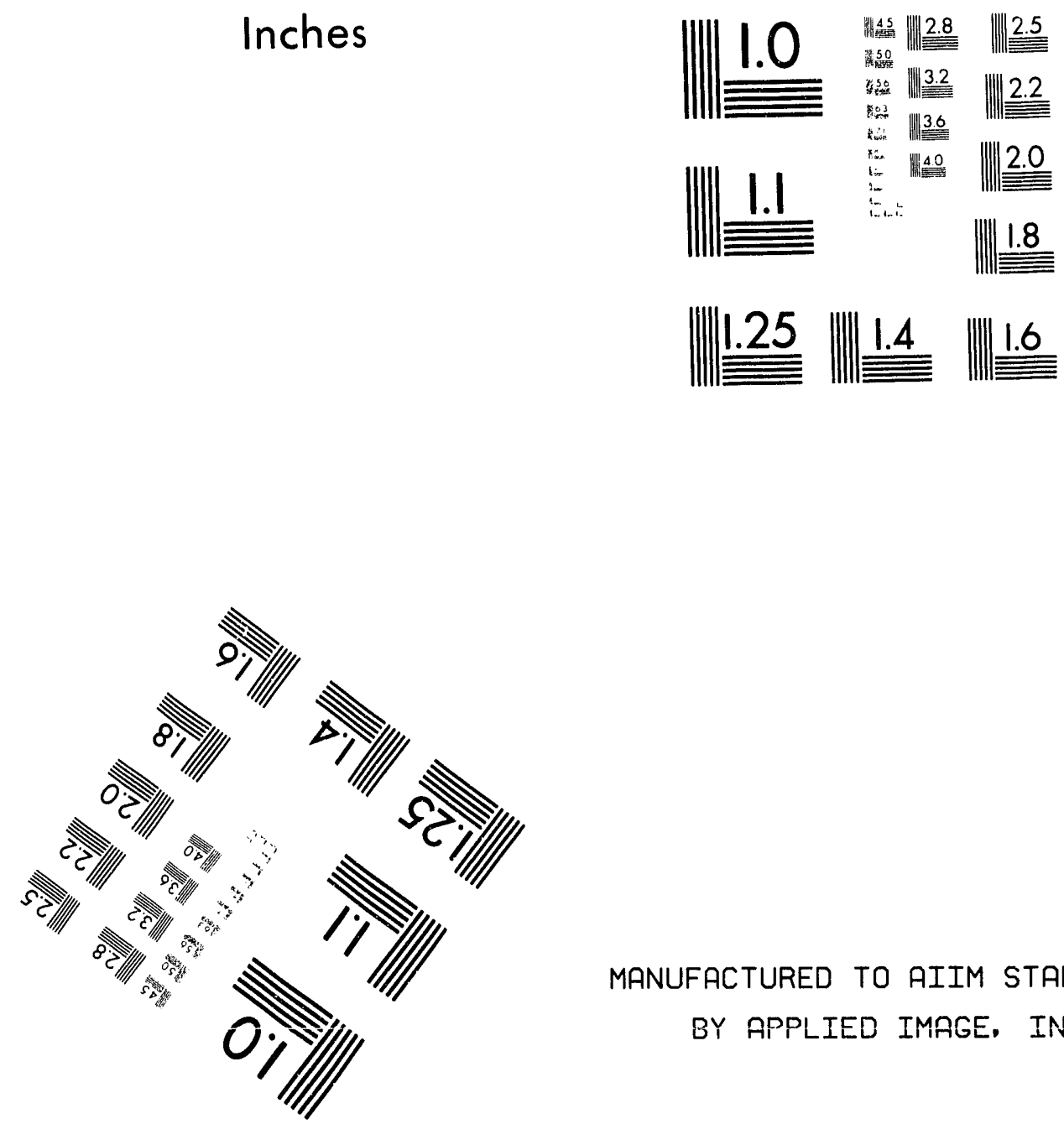

MANUFACTURED TO AIIM STANDARDS

BY APPLIED IMAGE. INC.

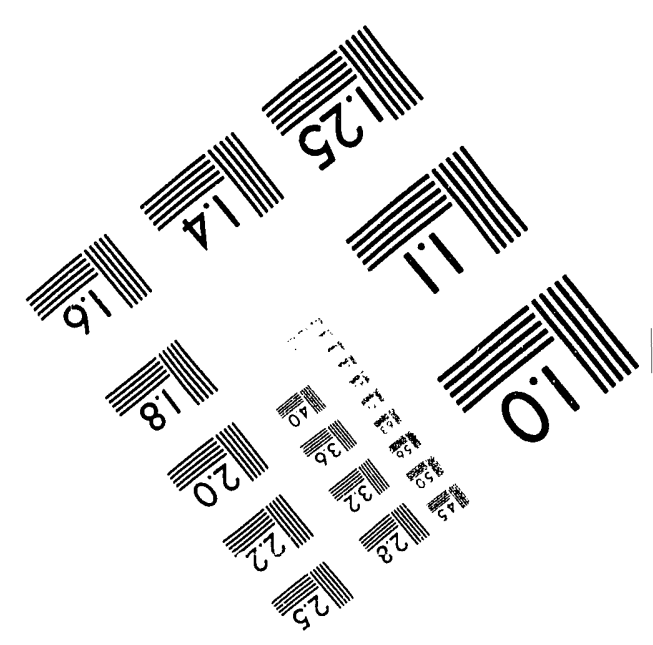



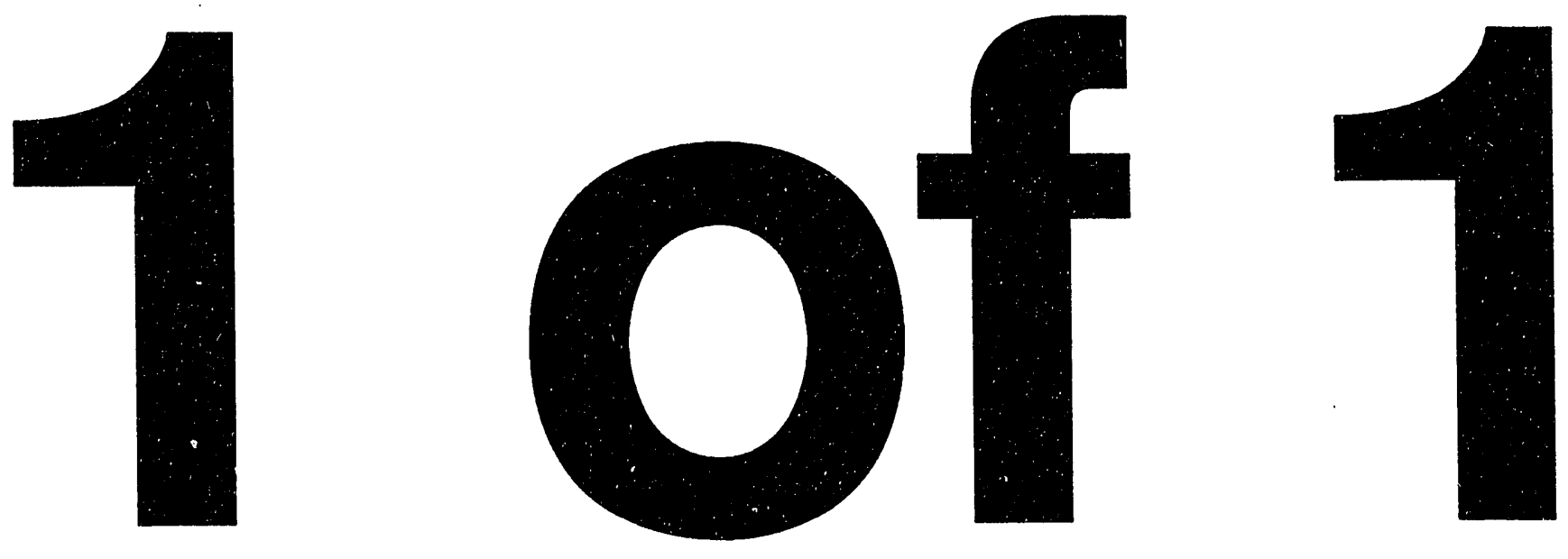


\section{ACE PROGRAM/UNIX USER MANUAL}

S. Kate Feng-Berman

January, 1993
RECEIVED
JUN 281993
OSTI

Research Supported by the OFFICE OF BASIC ENERGY SCIENCES

\section{NATIONAL SYNCHROTRON LIGHT SOURCE}

BROOKHAVEN NATIONAL LABORATORY ASSOCIATED UNIVERSITIES, INC.

Under Contract No DE-ACO2-76CH00016 with the

UNITED STATES DEPARTMENT OF ENERGY 


\section{I)ISCLAIMER}

This report was prepared as an account of work sponsored by an agency of the United States Government. Neither the United States Government nor any agency thereof nor any of their employees, nor any of their contractors, subcontractors, or their employees, makes any warranty, express or implied, or assumes any legal liability or responsibility for the accuracy, completeness, or usefulness of any information apparatus, product, or process disclosed, or represents that its use would not infringe privately owned rights. Reference herein to any specific commercial product, process or service by trade name, trademark, manufacturer, or otherwise, does not necessarily constitute or imply its endorsement, recommendation, or favoring by the United States Government or any agency, contractor or subcontractor thereof. The vifws and opinions of authors expressed herein do not necessarily state or retlect those of the United States Government or any agency, contractor or subeontractor thereof. 


\section{ACE PROGRAM/UNIX}

\section{USER MANUAL}

S. Kate Feng-Berman, NSLS, BNL

Jan. 12, 1993 


\section{Table of Contents}

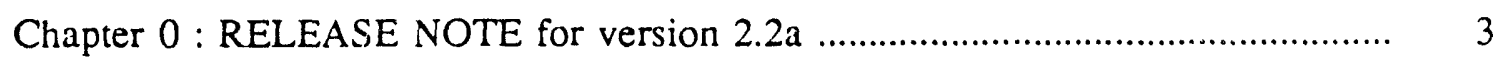

Chapter $1:$ How to use the ace program ? ............................................................ 7

1.0 : Introduction to the ace program .......................................................... 7

1.1 : Online command ........................................................................... 7

1.1.2 : Define a macro file ...................................................................... 9

1.2 : macro commands ........................................................................... 11

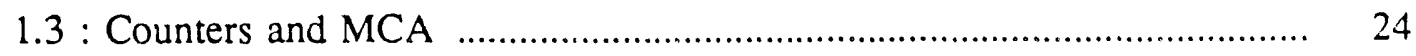

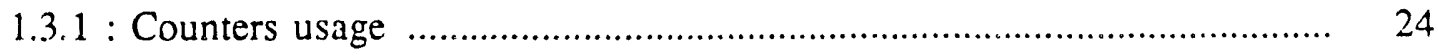

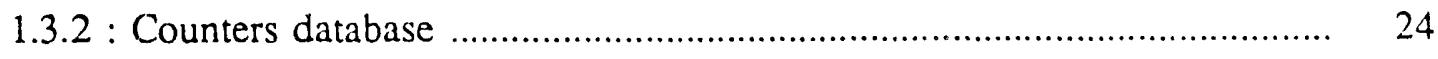

1.3 .3 : Feedback Counter Database ............................................................ 27

1.3.4 : MCA functions and macro command ................................................. 29

$1.4: \mathrm{X}$ window Interclient Communication ................................................... 33

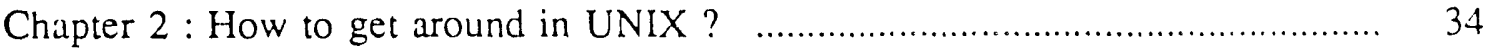




\section{CHAPTER 0}

\section{RELEASE NOTE for version 2.2a}

The version 2.2a program is called ace which stands for Automatic Control Environment. It's an updated version of the old go program, which includes a C-like syntax and some other new features. In this $\mathrm{C}$-like syntax, users do not have to declare any variable used in the macro definition. The updates are the followings:

1 The line editor command is 'edit' instead of 'def' which is used tc define a macro. Please reference section 1.2 : 'command edit' for the line editor 'edit', and section 1.1.2 and section 1.2: 'command def' about defining a macro.

2 The maximum number of arguments for a macro definition is ten which is represented by $\$ 1, \$ 2, \$ 3, \$ 4, \$ 5, \$ 6, \$ 7, \$ 8, \$ 9$, and $\$ 10$ respectively. The number of argument in a macro call is $\$ \#$, and the whole line of arguments is $\$ *$. Please read section 1.2 for command 'def'.

3 There are three options $(-\mathrm{f},-\mathrm{c},-\mathrm{m})$ when running ace program. They are : $-\mathrm{f}$ to load in a macro file, $-m$ ti, load in a motor diatabase file, and $-c$ to load in a counter database file. Besides, you can use a "dofile" command to load in macro files up to the maximum of five files at a time (please reference section 1.2 for the command 'dofile').

4 There are several new reserved variables : POS[], OUTNAME, CNT[]. You can use a 'print' or 'printf' command to print out expression lists to the desired output. The default output in the console. However, you can use '> "filename" or ' $>$ "filename"' to overwrite or append the result into a specified file instead of the standard output. Furthermore, you can use '> OUTNAME' or ' $>$ OUTNAME' to print anything to the data output filename specified by a 'newfile' or 'oldfile' command, or "disk.dat" file if the output filename is not specified by a 'newfile' or 'oldfile' comriand. This way, you can print a formatted string with variables such as motor position( POS[ ]), counts (CNT[]), a result of arithmetician operations, or even a comment to any output file. Please read section 1.2 command 'print' for details.

5 The 'datetime' command is replaced by the 'date()' command now. Please see command 'date()' for details.

6 The command 'tempfile' does not exist anymore since we can use the print command to print the result in any format we want into any file.

7 The format of a 'for' statement is changed to be C-language-like (i.e. nested statement is allowed). Please read section 1.2 command 'for' for details.

8 As I promised, now ace provides if-else, while, and do-while statements as in the $C$ programming language (i.e. nested statements are allowed). Please read section 1.2 command 'if-else' for the if-else statement, and command 'do-while' for a while statement. 
9 Since users can define their own variables (except those reserved variables mentioned above), the UNIX system command calls have to be preceded by a ' $\$$ ' symbol or use 'unix' command to avoid conflicts. Please read section 1.2 command unix.

10 In the 'PO' command, users can toggle between a linear(default) and log scale using the function key $\mathrm{F} 4$.

11 The online help command is ' $h$ ' instead of '?'.

12 The macro commands can be executed not only from a macro but also from the online input, like other online commands bu, cu, po, and so on. This is mentioned in section 1.1.

14 MCA support is much more user friendly after updating the MCA interactive graphical function, macro commands, and counter database. Please see section 1.3.1, 1.3.2 and 1.3.4 for those MCA features update.

15 Please do not forget to set the minimum speed on the E500 motors.

16 Macro command setup can be used to change the counter database also. Please see section 1.2 command setup and section 1.3.2 for counter database.

17 If a macro returns a value, please remember to use the '()' brackets to delimit the call by reference parameters which are seperated by comma. Please reference section section 1.1.2, and section 1.2: command return.

18 The format for ca_read, ca_write, ca_str, gpib_read, gpib_write are different now. Please reference section 1.2 for those commands.

Excuse me, the following is the 'to do' list.

1 Print out motor position in a different window.

2 Macro commands for MCA with (?) mark are not implemented yet! 


\section{RELEASE NOTE for version $2.0 \mathrm{~g}$}

1 If you want to rescale during a scan, type F4. Then, the program will ask you if you want the auto scaling. The auto scaling will optimize the plot automatically according to the real data.

2 Any macro filename with an extension '.mac' will not have to type the extension

3 The commands 'mvc', 'mvf', and 'mvp' support multi-motor alignment to move those motor to the center, curve-fitted peak, and peak position. Please read section 1.2 about command mvc, mvf, mvp.

\section{RELEASE NOTE for version 2.0a}

The following is changed in the new release.

1 The graphics will stay opened once it is opened until the user quits the program. When the graphics is opened, it can be focused in/out, moved and resized by the mouse in the same way as the other windows controlled by the window manager.

2 The feedback counters can be mixed in with any scan counter in any order in the counter database file. The maximum number of counters in the database file is ten. Any feedback counter can be one of the scan counter. (i.e. They could be using the same counter.)

3 The macro file commands 'countdwell','countdelay', 'countlimit', and 'countpreset' update not only the current counting but also the counter database file.

4 The macro file commands are zase sensitive. Most of them should be lowercase.

5 The movr command and read negg4.cnf command will not print the updated position and counts as a comment in the output file. If you want to print the update, you can add in a print statement right after the movr command or read counter command such as 'printf "pos \%12.4f cnts \%12.4f \%12.4f ", POS[in1], CNT[a], CNT[b]'.

6 The move commands for on line commands and macro file commands are identical. All the move commands of on line commands are changed from ' $m$ ' to ' $m v$ ' (i.e. $\mathrm{mr}, \mathrm{ma}, \mathrm{mm}, \mathrm{mp}, \mathrm{mc}, \mathrm{mf}$, and $\mathrm{mt}$ is changed to mvr, mva, mvm, mvp, mvc, mvf, and mvt, respectively). All the move commands for a macro file are changed form 'mov' to 'mv' (i.e. like movr, mova, movm, movp, movc, movf, and movt is changed to mvr, mva, mvm, mvp, mvc, mvf, and mvt, respectively).

7 The on line command for alignments or scans is the same as the macro file command. Please use 'align' or 'scan' for a single motor alignment or scan, and use 'alignm' or 'scanm' for a multi motor alignment or scan. Also, you can change the align(scan) parameters (i.e. start position, end position, and step size) from the 'align' or 'scan' command. The sign of the step size determines the alignment 


$$
-6-
$$

direction, but not the scan direction which is determined by the start and end position. Please see commands align, alignm, scan, scanm in section 1.2 for details.

8 In order to print a plot from 'PO' command, users must make sure the plotter number is set right in the counter database file. Please see section 1.3 .2 for counter database item 26 which setup the plotter number. 


\section{CHAPTER 1}

\section{How to use the ACE program?}

\subsection{Introduction to the ACE program}

First, you need an account in the UINIX. If you do not have one, please see your system administrator.

The program ace, which stands for Automatic Control Environment, is an updated version of go program, and incorporates most of its user interface. The users define all the macros with a C-like syntax in one or several files, and then load the files with a 'dofile' command (section 1.2: command dofile), or load them with the '-f' option while running the 'ace' program. To create those files, you can use any editor or the ace builtin line-editor-command 'edit' (section 1.2 : command edit). Please read section 1.1.2 and section 1.2: command def about defining a macro.

After login to your account, please type 'xinit' to start the $X$ window, and type 'cd $x \exp$ ' after $X$ window showing. This will bring you to the subdiretory 'xexp' under your account. Then you can type 'ace' with or without the '- $m$ ','-c', and '-f' options to start the program. When you type 'ace -m motor.cnf -c counter.cnf -f doall.mac', you automatically load a motor database (-m option), a counter database (-c option), and a macro definitions file (-f option). The ace program must be run under an $X$ window for graphics. There are two database files required, one is the motor database file (discussed in section 1.2 command setup) and another is the counter database file (discussed in section 1.3.2). It is suggested that you name thoses files with the extension '.cnf'. As to the data output file, you can give a filename without an extension, and then ace will create a new file with a lowest extension higher than the existing files extensions (available form .000 to .999 only). If you give a filename with an extension, the data will be appended to the file if the filename already exists.

\subsection{Online Command}

You can type ' $h$ ' for help at command input line to get the command summary. The online commands are the followings, plus the macro commands described in section 1.2 and section 1.3.4.

align: a motor ALIGNment scan. (align motor fleftoffset rightoffset stepsize filename)

alignm: Multi-motors ALIGNment scan. (alignm motor motor ......)

ba: $\quad$ motor dataBase file Append

bm: motor dataBase file Make (overwrite/create) 
bo: $\quad$ Open another motor dataBase file

bu: $\quad$ motor dataBase file Update

ca: $\quad$ Counter database file Append

ca_read: CAMAC Read data (example: ca_read(10, 17, 0))

ca_write: CAMAC Write data (example: ca_write $(10,25,8,4500)$ )

ca_str: CAMAC String data

$\mathrm{cm}$ : Counter database file Make (overwrite/create)

co: Open another counter dataBase file

cu: $\quad$ Counter database file Update

dofile: $\quad$ To read in macro definition file(s)

edit: A simple line editor to create/overwrite/append a macro file.

feedback: FeedBack by a certain number of times specified in the counter database.

feedbackc: Feedback Continuously.

gpib_read GPIB Read data ( example: print gpib_read(6,"IP"))

gpib_write: GPIB Write data (example: gpib_write $(6, " P 123 ")$ )

hi: $\quad$ HIstory listing of commands.

mva: $\quad$ Move to Absolute position (mva motorcode position)

mvm: $\quad$ Move Multiple motors at the same time. (mvm motor motor ....)

mvc: $\quad$ Move to beam intensity center position. (mvc motor [motor....] counter)

mvf: $\quad$ Move to beam intensity curve fit position. (mvf motor [motor ....] counter)

mvp: $\quad$ Move to beam intensity Peak position. (mvp motor [motor....] counter)

mvr: $\quad$ Move Relati ely to current position (mvr motorcode distance)

scan: A motors Scan from one position to another. (scan motor [start end stepsize filename])

scanm Multi-motors Scan from one position to another.

setup Setup motor or counter database file by the item number.

$\mathrm{p}$ : $\quad$ move the same distance as last $M R$ in $(+)$ direction

$\mathrm{n}$ : $\quad$ move the same distance as last MR in (-) direction

pa: $\quad$ Plot All scans of one data file in one plot

pca: $\quad$ MCA interactive graphical data control/collection/analysis with original data on all channels

pci: MCA interactive graphical data control/collection/analysis with all channels' data to be zero

po: $\quad$ Plot Old data

ri: $\quad$ Read Intensity from data source 
sp: $\quad$ Set/read unit position and pulse position

wa: Write the motor/counter information in ASCII file $\rightarrow$ motor_cnt.asc

q : Quit

!!: $\quad$ repeat last command

\subsubsection{Define a macro file}

A macro is like a function in a $C$ language, which consists of one to several commands. The command 'def' is used in a macro file to define a macro. Each macro file can have many macro definitions (each defined by a 'def' command). The format for the 'def' command is:

def macro_name'

The first line of the definition must include def macro name' followed with several lines of C-like-syntax commands, one command one line, or several commands in one line each separated by a semicolon (';'), and the macro definition is ended with another' at the end. The C-like-syntax command could be a C-like statement (i.e.:'if', 'while', 'for', etc.), a C-like variable assignment (i.e. energy=12.4), a C-like arithmetic operation (i.e. energy/=2* $\cos (1)$ ), a $C$-like input-output (i.e.: 'printf','getline'), a C-like string operation (i.e. 'sprintf', 'snlit'), an 'ace' builtin command (i.e.: 'mvr', 'scan'), or another defined macro command. All of them are described in section 1.2 for details.

Example:

def do_test 'unix("cd /usr/ul/feng/xexp")

if $(\$ \# !=2)$ \{ printf("Usage: do_test distance counter/n"); 'exit \}

mvr theta $\$ 1$; set_it 2.5

for $(i=0 ; i<2 ; i++\overline{)}$;

read $\$ 2$

$\operatorname{mvr}$ axe $2 * i$

\}'

The first line starts the definition of 'do_test' macro, and changes the current directory to /usr/ul/feng/xexp so that the input and output file will be at /usr/ul/feng/xexp directory (this is a UNIX system command). The second line checks if the macro is called with the right arguments. The third line has two commands. The first command moves motor theta relatively according to the first argument, followed by a ';' as a command separator and the second command calls another defined macro command 'set_it' with one argument '2.5'. The line four to line seven consist of a loop which reads the counter specified by the second argument, and moves motor axe relatively two units in first loop and four units in second loop. When a function is called, the passed parameters can be a defined variable like the above example in line six, which uses loop number ' $i$ ' to decide how much distance to move motor 'axe'. Usually, the macro name does not have an extension, while the macro files have a '.mac' extension. 
If a macro returns a value, please remember to use the '()' brackets to delimit the call by reference parameters which are seperated by comma.

Example:

def calc_it,

\# This function retunrs a value

return $(\cos (\$ 1)+\$ 2)$ '

In the above example, the fucntion calc it is defined as another arithmatic operation which ran be called like other builtin arithmatic operation (i.e. printf(" $\cos (1.2)+3.5=$ $\% 12.4 \mathrm{fn}$ ", calc_it $(1.2,3.5))$; ). In the above function, '\#' is used to start a line with a comment. 


\section{2 macro commands}

The following commands are macro commands appearing in an alphabetical order, and they are case sensitive. Regarding the macro commands for the MCA devices, please reference section 1.3.4 for details. In the format description, all arguments enclosed by [ ] are optional, but they have to be given in the specified order if used.

Command : align

Purpose : If the step size is greater than zero, it performs an alignment scan from the [current position - scan left] to the [current position + scan right], and then returns to the current position. If the step size is smaller than zero, it reverses the alignment from the [current position + scan right ] to the [current position - scan left], and then returns to the current position.

Format : align MotorCode [leftoffset rightoffset stepsize filename]

Example : align theta

: align theta 320.5 (It changes align left, right, and step size in motor database file first, then does alignment with the new argument)

Note: If the options within [] are specified, they have to be given in the above order, and the database file will be updated on the specified motor.

$\begin{array}{ll}\text { Command } & : \text { alignm } \\ \text { Purpose } & : \text { It performs a multi-motors' alignment } \\ \text { Format } & : \text { alignm MotorCode MotorCode ............ } \\ \text { Example } & : \text { alignm theta ome pm }\end{array}$

Command : break

Purpose : It terminates the innermost enclosing 'while' or 'for' loop. It is like a C language 'break' statement.

Format : break

Command : ca_read

Purpose : It gets data from a CAMAC crate.

Format : ca_read(slot\#, function, address) 
Example 1 : pri.uf("The data read from CAMAC slot 2, func 0,addr 0 is \%ldn",ca_read $(2,0,0)$ );

Example 2 ret_value $=$ ca_read $(10,25,8)$

Command : ca_str

Purpose : It sends a command string to a CAMAC crate.

Example 1 : ca_str("z")

Example 2 : ca_str("i")

Note : Only the following three commands are supported:
$z:$ itialization
i : inhibit
c : clear inhibit
Command : ca_write
Purpose $\quad:$ It sends data to a CAMAC crate
Format : ca_write(slot\#, function, address, data)
Example : ca_write $(2,16,0,100)$
Command : continue
Purpose : It passes control to the loop-test portion of the innermost enclosing 'while' or 'for' loop.
Format : continue
Command : countdelay
Purpose : It delays counting after motor finishing moving.
Format : countdelay DelayTime (in seconds)
Example : countdelay 10
Note It not only changes the counting delay time but also updates the counter database file.

Command : countlimit

Purpose : It setups the counter low limit 
Format : countlimit \#

Example : countlimit 1000

Note : It not only changes the counter low limit but also updates the counter database file.

Command : countdwell

Purpose $\quad:$ It setups the count dwell time in second.

Format : countdwell Time Base (dwell= Time $* 10$ (Base))

Example : countdwell 12 (dwell $=100)$

Note : : It not only changes the counting time but also updates the counter database file.

Command : countpreset

Purpose : It setups the counter preset number

Format : countpreset preset\#

Example : countpreset 1000

Note : It not only changes the counting preset value but also updates the counter database file.

Command : date()

Purpose : The date() function returns the current date and time as a string.

Format : date()

Example : print "\#"date() > O NAME (print current date and time as a comment in the current specified output file).

Command : def

Purpose : It defines a macro with arguments. As of today, the maximum number of arguments for a macro is ten, which is represented by $\$ 1, \$ 2, \$ 3, \$ 4$, $\$ 5, \$ 6, \$ 7, \$ 8, \$ 9$, and $\$ 10$ respectively. The number of argument in a macro call is $\$ \#$, and the whole line of arguments is $\$ *$.

Format : def macro_name,

Note $\quad$ A macro is defined within a pair of apostrophe ('....').

Example def scanloop'

if $(\$ \# !=5)$ 


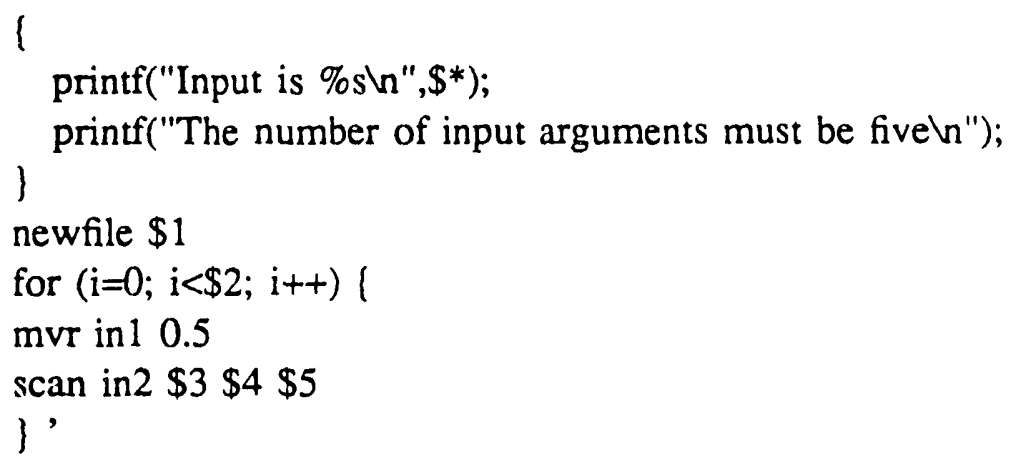

After building the macro file called 'scanloop', you can call it with varied arguments like 'scanloop scan_test 25.0 10.0 1.0'.

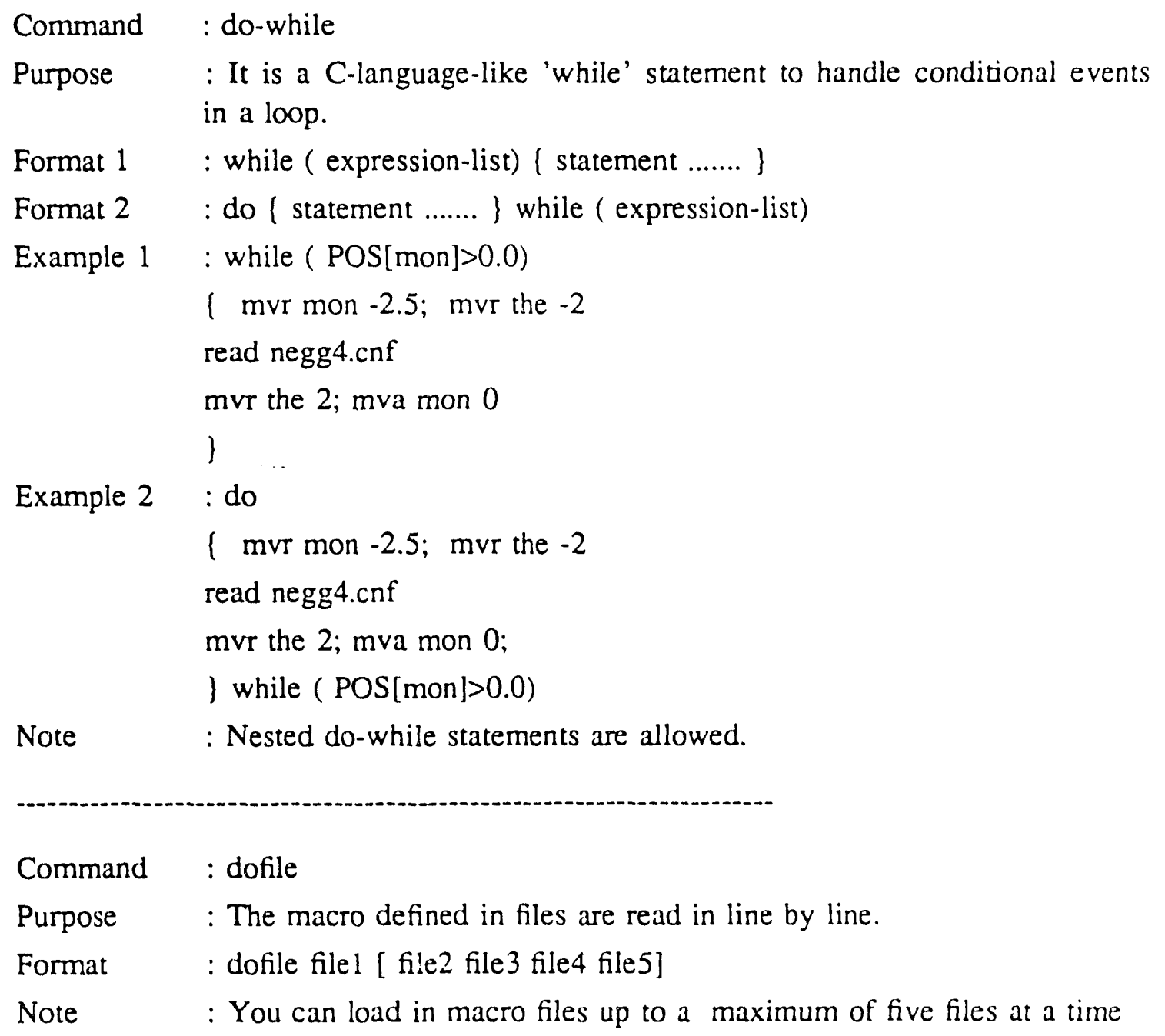




\section{Command : edit}

Purpose : It is a simple line editor which can create, append, or overwrite a macro file without using the other complicated editor. It is a line editor. That is to say, you can only modify the current line, but not the previous lines.

Format : edit filename

Note

: After you type edit filename followed with an ENTER, if the filename already existed, it will ask you whether you want to quit, overwrite or append the file. Then you have to start the editing with def macro_name', and end the editor with another'.

Command : exit

Purpose : It terminates the execution of the current macro, and jumps control back to command level. It is a C-language-like exit statement.

Format : exit

Command : feedback

Purpose : It performs a feedback function to tune up a system. It will work only if the feedback preset counter and up counter are setup and enabled for feedback. Please read section 1.3.3 for feedback counter setups.

Forma: 1 : feedbackc (It performs a feedback continuously. Type ${ }^{\wedge} \mathrm{C}$ to exit.)

Format 2 : feedback ( It performs a feedback by a certain number of times which is specified in the feedback up counter database item 29: tuneup times/feedback.)

$\begin{array}{ll}\text { Command } & \text { : for } \\ \text { Purpose } & \text { : It is a C-language-like 'for' statement to do things in a loop. } \\ \text { Format } & \text { for }(v a r=0 ; \operatorname{var}<10 ; \operatorname{var}+=2) \\ & \{ \\ & \text { xxxx } \\ & \} \\ \text { Example } & : \text { for }(\mathrm{i}=1 ; \mathrm{i}<6 ; \mathrm{i}++) \\ & \{\operatorname{mvr} \operatorname{mon} 2 * \mathrm{i} ; \operatorname{mvr} \text { in } 14.6 ; \\ & \text { read negg4.cnf }\end{array}$


for $(x=1 ; x<3 ; x++) m v r$ in $3 \cos (x)$;

\}

Note $\quad$ : Nested 'for' statement is allowed.

Command : gpib_read

Purpose : It sends a read command through GPIB to a device, and then print the read string on the stdout.

Format : gpib_read(dev, command)

Example 1 : printf("The motor pulse position is \%ldn", gpib_read(6,"IF"));

Example 2 : read_pos=-gpib_read(6,"IP");

Command : gpib_sread

Purpose : It does the same thing as gpib_read except it uses slow handshake sequence to read string one byte by one byte. It is used for a device with a slow handshake timing.

Format : gpib_sread(dev\#, command)

Example : read_value $=\operatorname{gpib} \_\operatorname{sread}\left(3,{ }^{\prime} R C^{\prime \prime}\right) / 3.0$;

Command : gpib_write

Purpose : It sends a write command through GPIB to a device

Format : gpib_write(dev\#, command)

Example : gpib_write $(10,099999)$

: gpib_write( 6, "P20 X-1000 Y1000")

Command : if-else

Purpose : It is a C-language-like 'if' statement to handle conditional events.

Format 1 if (expression-list) \{ statement ...... \}

Format 2 if (expression-list) \{ statement ...... $\}$ else $\{$ statement .... $\}$

Example $\quad:$ distance $=5.0$; if ( POS [the $]>10.0$ )

( mvr mon 2.5; distance- $=2.4$;

read negg4.cnf; printf("counter red counts \%12.4fn",CNT[red])

\} 


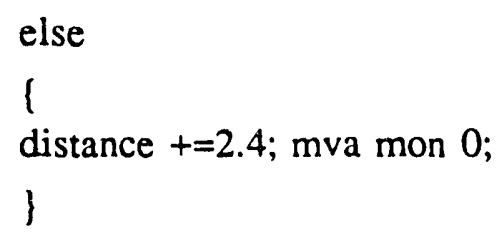

Note $\quad$ : Nested if-else statements are allowed.

Command : index

Format index $(s, t)$

Purpose It returns position of string $t$ in $s, 0$ if not present.

Example : index("banana","an")

( It returns 2.)

$\begin{array}{ll}\text { Command } & \text { : length } \\ \text { Format } & \text { length(s) } \\ \text { Purpose } & \text { It returns length of s. } \\ \text { Example } & \text { : length("bananna") } \\ & \text { (It returns } 7 .)\end{array}$

Command : mva

Purpose : It performs an absolute move.

Format : mva MotorCode Position

Example : mva theta 0

$\begin{array}{ll}\text { Command } & : \text { mvr } \\ \text { Purpose } & : \text { It performs a relative move. } \\ \text { Format } & : \text { mvr MotorCode Distance } \\ \text { Example } & : \text { mvr theta } 45\end{array}$

Command : mvm

Purpose : It performs a multi-motors' relative motion at the same time. (All the motors are moved relatively to their current positions) 
Format : : mvm MotorCode Distance MotorCode Distance MotorCode Distance

Example : mvm to 10 t2 5 al 10

Command : mvp

Purpose : It does alignment scans and moves those scan motors to the beam Peak position. The number of alignment motors could be more than one.

Format : mvp MotorCode [MotorCode ......] CounterCode [filename]

Example : mvp the mon ind roi

Note: The filename is an option. If it is specified, a full filename must be given. If it is not specified, it is the default output file specified by 'oldfile' or 'newfile'.

Command : mvc

Purpose : It does alignment scans and moves those scan motors to the beam peak CENTER position. The number of alignment motors could be more than one.

Format $\quad$ :mvc MotorCode [MotorCude ....] CounterCode

Example $\quad: \mathrm{mvc}$ the $\mathrm{cnt}$

: mvc the ome cnt

Command : mvf

Purpose : It does alignment scans and moves those scan motors to the beam curve-fitted peak position. The ..umber of alignment motors could be more than one.

Format : mvf MotorCode [MotorCode........] CounterCode

Example : mvf theta cnt

Command : newfile

Purpose : It specifies a data filename for the output.

It is required before data scan to specify the data output filename, otherwise data will be written to the programmed default data file 'disk.dat'. If the specified filename has no extension, it will open a new file with an extension whose number is one higher than the existing highest 
extension. Otherwise, the new data will be written to the end of the specified file.

\section{Format : newfile Filename}

Example : newfile test.002 (The output file is test.002)

Example : newfile test (If the highest extension in the current directory for test is .010 , then the output file is test.011)

Command : oldfile

Purpose : It specifies an old data filename for the output.

The given filename should not come with an extension. It will look for the current existing files with the same filename and with the highest extension number. The new data will be appended to the old file.

Format : oldfile Filename

Example : oldfile test 1

Command : print (or printf)

Purpose : The command 'print' prints an expression list on an output, while the command 'printf' prints a formatted expression list on an output.

The default output is the console. You can use '> OUTNAME' or '>> OUTNAME' to print anything to the data output filename specified by 'newfile' or 'oldfile' command, or "disk.dat" file if the output filename is not specified by 'newfile' or 'oldfile' command. You can further use '> "filename"' or '>> "filename"' to overwrite or append the result into a specified file instead of the standard output. This way, you can print a formatted string with variables such as motor position( POS[ ]), counts (CNT[]), a result of arithmetician operations, or even a comment to any output file. Please remember to quote a string and the filename with " ". There are several new builtin variables : POS[]( for motor position), OUTNAME (for data output filename), CNT[] (for counter data), the motor code and counter code read from the database file. All the builtin variables except 'OUTNAME' can not be specified a value by user. The motor-code variable or counter-code variable whose numeric value is the order of the motor or counter appearing in the database file, and motors, counters must not have the same name. In order to get the right numeric value for the motor-code variable or the counter-code variable, it is forbidden to use a number as the first character of the code, and the maximum number of characters for a code is three. The index for POS[] is the motor-code variable and the index for CNT[] is the counter-code variable. The arithmetic functions are as the followings: 


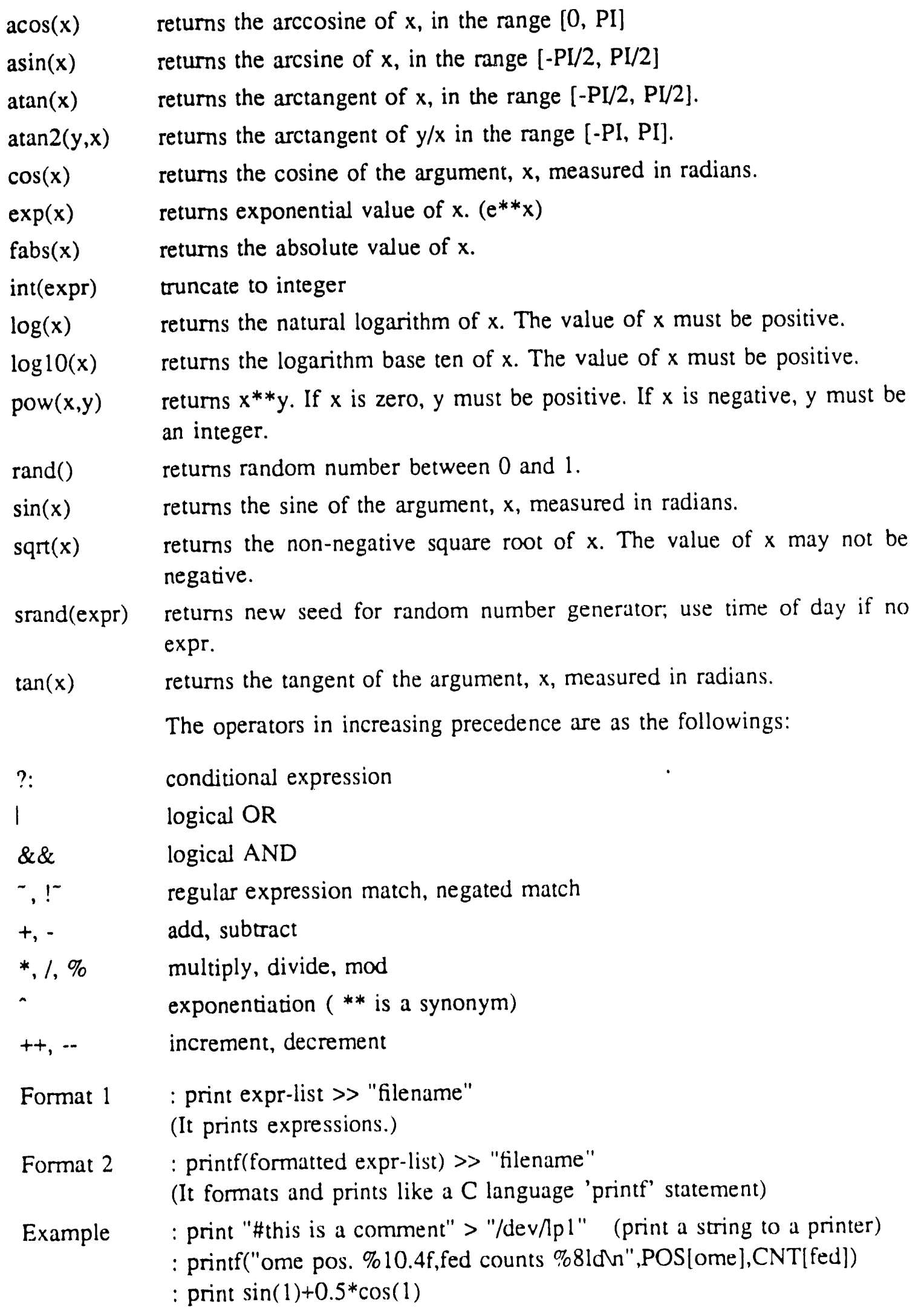




$\begin{array}{ll}\text { Command } & \text { : read } \\ \text { Purpose } & : \text { It reads intensities from a counter source. } \\ \text { Format } & : \text { read CounterSource } \\ \text { Example } & : \text { read egg4.cnf }\end{array}$

Command : return

Purpose : It returns a value from a defined macro.

Format : return(value)

Example $\quad:$ return $(\sin (3.0) / 2.0)$

Command $9:$ scan

Purpose : It performs a scan from a start position to an end position. Unlike an alignment, the direction of a scan is not determined by the sign of the step size, but the start position and end position. scan.

Format : scan MotorCode [start end stepsize filename]

Example : scan theta

: scan theta 12.02 .01 .0 ( It changes scan start position, end position, and step size in motor database file first, then does alignment with the new argument )

Note: If the option within [] is specified, they have to be giver in order, and the database file will be updated on the specified motor.

$\begin{array}{ll}\text { Command } & \text { : scanm } \\ \text { Purpose } & \text { : It performs a multi-motors' scan } \\ \text { Format } & : \text { scanm MotorCode MotorCode } \\ \text { Example } & \text { : scanm theta pm }\end{array}$

Command : setup

Purpose : It updates the database parameters of the specified motor or counter.

Format 1 : setup MotorCode item\# value item\# value..........e.t.c (update motor database)

Format 2 : setup CounterCode item\# value item\# value.........,e.t.c (update counter database) 
Example : setup theta 50.0191000

Please reference section 1.3.2 for counter database parameters. As to the item\# for each motor database parameter, it is listed as followings.

1: Driver Type

(ASCII string): The current supported motor driver type are 'MMC' for a GPIB system, and 'E500', 'DACO' ( [-2.5.2.5] volts : 12 bits), 'DACl' ([0,5] volts : 12 bits $)$, 'DAC2' $([0,10]$ volts : 12 bits), 'DAC3' ([-5,5] volts : 12 bits), 'DAC4' ([$10,10]$ volts : 12 bits $)$, 'DAC5' ([0,10] volts : 16 bits) for a CAMAC system. The drivers 'DAC0' to 'DAC4' are designed for Joerger model DAC-16, which is a 16 channel, 12-bit-resolution digital to analog converter. The driver 'DAC5' is designed for Joerger model D/A-16, which is a dual channel 16-bit-resolution digital to analog converter. Monochrometer motor with nonlinear function between pulse position and unit (eV) position can be implemented by appending ' $x 25 \mathrm{~m}$ ' ( for beamline X25) or ' $\mathrm{x} 19 \mathrm{~m}$ ' (for beamline $\mathrm{X} 1 \overline{9}$ ) after the driver type like 'mmc' or 'e500' (i.e.: e500_x $25 \mathrm{~m}$, or mmc_ $\times 25 \mathrm{~m}$ ). The scale factor and calibration nummber vary from different kinds of crystals used.

2: Motor Code (1-3 char. ): A short name used as a code in the motor commands.

3: Motion Name (1-20 char. ): A description of the motor function.

4: Slot Number (Integer ): The slot number on the CAMAC crate, or the device number on the GPIB board.

5: Motor Address (Integer ): Motor channel number or DAC channel module.

6: Unit Name (ASCII string): e.x. mm,inch,degree,...,etc. For DAC module, it is volts or voltages.

7: Minimum Speed ( pulses/sec ): The starting speed.

8: Maximum Speed (pulses/sec ): The peak speed. If a DAC module is used, it is the number of bit pulses sent out on each ramp step (i.e. voltage change/ramp step).

9: Acc. Rate

( pulses/S/S ): The acceleration rate. If a DAC module is used, it is the delay time in milliseconds between each voltage change ( ramp step).

10: Pulse Position (long int ): The motor position in unit of pulses.

11: Backlash

(Integer ): The number of pulses for backlash. If the backlash value is positive, a backlash occurs only when the motor is moving in a positive direction. If the backlash value is negative, a backlash occurs only when the motor is moving in a negarive direction. 
12: BacklashOn

13: ActiveOn

14: Disabled

15: Scale Factor

16: Lower Limit

17: Upper Limit

18: Current Position

19: Align Left

20: Align Right

21: Step Size
( $\mathrm{Y} / \mathrm{N} \quad$ ): Enable/disable the backlash occurrence.

( $1 / 0=$ on/off ): Set the motor to be active or not. If the motor is set active, its position will be printed each time before a command input prompt, and its database will be in the data output file.

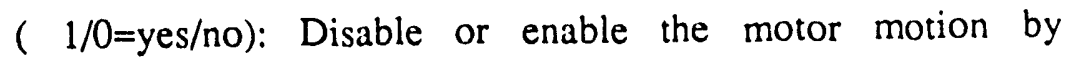
software.

( pulses/unit): It is the number of pulses per unit. If DAC module is used, it is hard-coded (set by the computer), and the unit is bits/voltage.

(Units ): Software low limit.

(Units ): Software high limit.

(Units ): The motor position in unit.

(Units ): The alignment distance from the current motor position to the left. It is always a positive value.

(Units ): The alignment distance from the current motor position to the right. It is always a positive value.

( Units ): The step size in unit for the motor alignment and motor scan. The sign of the step size determines the direction of the alignment, but not the direction of the scan. If it is a negative value, then the motor aligns from the right to left.

22: scan Start Pos. (Units ): The motor scan start position in unit.

23: Scan End Pos. (Units ): The motor scan end position in unit.

24: Data Source (ASCII string): The name of a counter database file which was set with all the counters' parameters for the motor scan.

25: Calibration no. (float): The calibration constant to be used in the monochrometer motor.

(1/0=yes/no ): Enable/disable the feedback during scan or alignment points.

Command : sprintf

Format sprintf(fmt,expr-list)

Purpose It prints expr-list according to $\mathrm{fmt}$, and returns resulting string.

Example $\quad$ str $=$ sprintf("Mo \%s pos \%12.4f",th2, POS[th2]) 
Command : substr

Format substr(s,i,n)

Purpose It returns $n$-char substring of $s$ starting at $i$; if $n$ omitted, use rest of $s$.

Example str=substr("North American", 1,5)

( It returns "North".)

Command : uniix

Purpose $\quad:$ It is used to call a unix system command.

Format 1 · unix("command")

Format 2 : \$command

Note 1 : You can define the often used system command in a macro file.

Note 2 To avoid conflicts, please do not define any variable starting with ' $\$$ ' or a digit.

Example 1 : unix("ls -l")

Example 2 : \$ls -1

Example 3 : def 1 'unix("ls -1 " \$*)' (make sure 'l' command is not defined yet) 


\subsection{Counters and MCA}

MCA is a multi channel analyzer. In the ace program, we can use the Region of Interest (called ROI) of the MCA as a counter. Besides, there are interactive graphical functions and macro commands for the MCA device to do spectrum anslysis and acquisition. The 1.3 subsections discussed all the details about the counters and MCA.

\subsubsection{Counters usage}

The data source in the motor database is actually a counter database filename. You can have different counter setups with different counter database files. There are three kinds of counter database supported. They are called egg4.cnf which is a setup for a EG\&G 874 counter module, negg4.cnf which is a setup for a EG\&G 974 counter module, and kcnt.cnf which is a setup for a Kinetic counter module 3640, or module 3610-L2A. Under each setup, you can have some counters set up from the ROI (Region Of Intreset) of an 'MCA'.

To use the kinetic counter, you need to use model 3640, or model 3610-L2A, a real time clock, and/or a clock generator. Then you should connect END signal of real time clock to INHIBIT signal of counter, and loop the PRESET OUT of real time clock to its START signal. If you want an external clock for the real time clock, take the signal from a clock generator to IN-A of real time clock.

When you set up the ROI of the 'MCA' as a counter, you have to gate the 'GATE' input on the back of MCA module with either the 'INTERVAL' output from an EG\&G counter or the 'BUSY' output from a Kinetic counter to make MCA counting synchronized with either EG\&G counters or Kinetic counters. Hence, the MCA ROI counters never exist alone in a counter database file. They are one of counters in either an EG\&G counter database file or a Kinetic counter database file. Besides, to get the sum of serveral ROI, please do the following: 1. Make sure the LTB jumper is at the 'ROI OUT' position, 2. Feed the 'LTB' signal from the back panel of the 'mca' card into a EG\&G counter or a Kinetic counter, 3. set those ROI number to be between 128 and 255. Furthermore, if you set the ROI number to be 0 in one of the ROI counter, you will get the counts of all channels in current group.

So far, this program supports up to ten counters in the database file. Any counter could be setup either for a scan or for a feedback. The feedback counter setup is discussed in section 1.3.3.

\subsubsection{Counter database}

The scan counters in the database file has the following parameters:

1: Driver Type
(ASCII string): The scan counters' driver type could be egg4.cnf, negg4.cnf, kent.cnf, and maa. When the driver type is egg4.cnf, negg4.cnf, or kcnt.cnf, it is also a counter database filename, 
which is the data source parameter in the motor database file. When mca is a driver type of a counter, other counters in the same database file must include the other driver type like 'egg4.cnf', 'negg4.cnf', or 'kcnt.cnf', because 'mca' device is gated by other type of counter for counting synchronization. The follwing database parameters which are marked with a '*' at the and are those needed for 'mca' driver type. They are the slot number (group number), counter channel (ROI number), counter time (ROI's start channel), counter base (ROI's end channel), and so on.

\section{2: Counter Code*}

3: Counter Name*

4: Slot Number*

5: Counter Channel*

6: Unit Name

7: Feedback?/Scan counter?

8: Counting in a scan *

9: Plot counter data *

10: Plot Data Symbol ?

11: Timer scan/Preset count?
( 1-3 char. ): A short name used as a code in the counter command.

( 1-20 char. ): A description of the counter function.

( Integer ): The counter slot number on the CAMAC, or the device number on the GPIB board. If 'mca' is the driver type, it is the MCA group number.

( Integer ): The counter channel number. If 'mca' is the driver type, it is the ROI's number.

(ASCII string): It is counts for the count-preset mode, or unit of time (i.e. second) for the timer mode.

$(1 / 0: \mathrm{Y} / \mathrm{N}):$ It specifies whether this counter is used in a feedback or a scan. The following parameters are for scan counters, and the parameters for feedback counters will be explained in the following section 1.3.3.

( $1 / 0: Y / N)$ : It is a flag for a counter's counting. If it is ' 0 ', the counter's counts will not be outputted.

( $1 / 0: Y / N):$ It specifies whether or not to plot a counter's data.

( $1 / 0: \mathrm{Y} / \mathrm{N})$ : It specifies whether or not to plot each counter data point with the associated symbol.

$(1 / 0 \quad)$ : It specifies whether it is a timer mode or preset-count mode. 
12: Preset counter ?

13: Beam monitor?

14: Count low limit

15: Counter Preset

16: Counter Time*

17: Counter Base*

18: Counter Delay
( $1 / 0: \mathrm{Y} / \mathrm{N}$ ): You should specify one of the counters as a preset counter which specifies the value of preset counts if you are using a presetcount mode for Kinetic module. If you did not give one, the default is the first counter for the scan and none for the feedback.

$(1 / 0: Y / N)$ : If the counter's count is used as a beam monitor, then please input ' 1 ' here.

(Integer ): It is used as a value to monitor beam intensity so that the counter will not take the counts if there is a beam dump or a low beam. It is valid only on the beam monitor counter.

( long/counts): It is used if the counter is set for a preset mode. Usually a negative value, it is valid only on the preset counter. The preset value for the Kinetic counter has the following restrictions. If the absolute value of preset is smaller than $65536(2 * * 16)$, it could be any value. If the absolute value of preset is in the range of $\left[65536,524,288\left(2^{* *} 19\right)\right]$, it must be a multiple of 8. If the absolute value of preset is in the range of $[524,288(2 * * 19),(2 * * 22)]$, it must be a multiple of 64 . If the absolute value of preset is in the range of $[(2 * * 22),(2 * * 25)]$, it must be a multiple of 512. If the absolute value of preset is in the range of $\left[\left(2^{* *} 25\right),(2 * * 28)\right]$, it must be a multiple of 4096. If the absolute value of preset is in the range of $[(2 * * 28),(2 * * 32))$, it must be a multiple of 32768 .

(Integer ): It is used for a EG\&G counter in timer mode (time $=M *\left(10^{* *} N\right)$, where $M$ is the counting time, $\mathrm{N}$ is the counting base). If 'mca' is the driver type, it is the start channel of the ROI number.

(Integer ): It is used for a EG\&G counter in timer mode (time $=M *\left(10^{* *} N\right)$, where $M$ is the counting time, $\mathrm{N}$ is the counting base). If 'mca' is the driver type, it is the end channel of the ROI number.

( seconds ): The delay time in counting after motor's motion. 
26: Plotter ? (0,1: Epson. 2,3,4,5: laser)

It specifies a plotter to be an Epson printer $(0,1)$, a local laser printer $(2,3)$, or the laser printer located at the hall of $R \& D$ group $(4,5)$. It should be set on the first counter. For the laser printer, plotter 2 or 4 generates a landscape (horizontal) output, while plotter 3 or 5 generates a portrait (vertical) output. If a Laser printer is chosen to print an image, please click the mouse in the desired window when the cursor becomes a cross.

28: Real time clock slot

It is the CAMAC slot number of the real time clock (module 3640, or 3610-L2A) if a Kinetic counter is used.

\subsubsection{Feedback Counter Database}

The feedback counters could be one of the scan counters. To do the feedback, you have to set up a preset down counter, an up counter, and optionally a normalizing counter. Besides, if you want to enable the feedback tuneup during a scan, you have to set the enable flag to be one on the feedback preset down counter. The normalizer is used to normalize the beam intensity in case there is too much change in the beam intensity as time goes on. Also the normalizer counter can be used to monitor the beam intensity. If the normalizing counter is used, the actual counts from the up counter and the normalizing counter will be normalized by the previous normalizer's counts. The previous normalizer's counts can be setup by giving any reference value in the up counter. The feedback counters in the database file has the following parameters:

\section{1: Driver Type \\ 2: Counter Code \\ 3: Counter Name}

4: Slot Number

5: Counter Channel

6: Unit Name

7: Feedback?/Scan counter?
(ASCII string): It is the counter database filename.

( $1-3$ char. ): A short name used as a code in the counter command.

( 1-20 char. ): A description of the counter function.

(Integer ): The counter slot number on the CAMAC, or the device number on the GPIB board.

( Integer ): The counter channel number.

(ASCII string): It is counts for the count-preset mode, or unit of time (i.e. second) for the timer mode.

( $1 / 0: Y / N)$ : It specifies whether this count. Is used in a feedback or a scan. 
8: Feedback up counter?

9: Feedback enabled?

10: Feedback mode?

11: Timer mode/Preset count?

12: Preset down counter?

13: Feedback Normalizer?

14: Count low limit

15: Feedback preset

16: Counter Time

17: Counter Base
( $1 / 0: \mathrm{Y} / \mathrm{N})$ : This is the counter which compares its counts with the feedback reference value used for tuneup. If this counter is not set in the database file, the feedback will do nothing.

( $1 / 0: Y / N)$ : Specified in the preset counter, it enables (1) or disables ( 0 ) the feedback capability of the program. To further enable a feedback during a scan, you have to enable the feedback in the scan motor database. ( item 26 of the motor database )

(0:ref, 1:max,2:min): This is specified in the preset counter. In the reference mode, the up counter is compared with the multiplication of reference value (parameter 14) and offset (parameter 23). In the max. mode, the up counter is compared with its last count to get the maximum counts. In the min. mode, the up counter is compared with its last count to get the minimum counts.

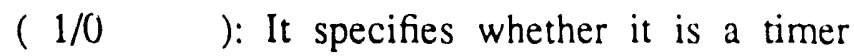
mode or preset-count mode.

( $1 / 0: \mathrm{Y} / \mathrm{N})$ : Used as a preset counter for tuneup counting period, it has to be set in the counter database file to do the feedback.

( $1 / 0: \mathrm{Y} / \mathrm{N}$ ): If you want to normalize the beam intensity, you should set a counter as a normalizer. After set, it will start counting in the preset counting period to get the normalized counts.

( Integer ): Specified in the feedback normalizer counter, it sets the normalizer's low count limit so that the feedback will not happen if the normalizer counts is lower the low limit.

(long/counts): It is the preset value for the feedback preset counter. Usually a negative value, it is specified in the preset counter for preset-count mode.

( Integer ): Specified in the preset down counter, it is used for a EG\&G counter in timer mode. (time $=M *\left(10^{* *} N\right)$, where $M$ is the counter time, $\mathrm{N}$ is the counter base)

( Integer ): Specified in the preset down counter, it is used for a EG\&G counter in timer mode. (time $=M^{*}\left(10^{* *} N\right)$, where $M$ is the counter 
18: Feedback loop delay

23: Feedback offset

24: Feedback motor code

27: Feedback reference

28: Scan points/feedback

29: Tuneup times/feedback time, $\mathrm{N}$ is the counter base)

( seconds ): Specified in the feedback up counter, it is the number of delaying seconds in the feedback loop between each tuneup.

$(0.0-1.0)$ : Specified in the feedback up counter, it is the offset factor (between 0.0 and 1.0) from the reference value.

(up counter ): Specified in the feedback up counter, it is the code of the motor which controls the feedback function.

(up counter ): Specified in the feedback up counter, it is the absolute value of feedback-upcounter's counts for tuneup. Whenever the reference value is setup in the up counter, it will change the normalizing counts also (if the normalizer exists!).

( Integer ): Specified in the feedback up counter, it is the number of scan points for each feedback action.

( Integer ): Specified in the feedback up counter, it is the number of tune up in each feedback loop.

\subsubsection{MCA functions and macro command}

The MCA interactive graphical functions will become available if you type 'pca' or 'pci' at the command line. Besides, if you set up one of the counters using MCA's ROI (section 1.3.2), the interactive graphical function will appear automatically when you start the ace program unless all the counters using MCA's ROI are disabled for plotting. The interactive graphical functions are quite compatiable with those under the DOS Operating System except some functions are optimized for being user friendly.

The followings are macro file commands to control MCA (Multi-Channel Analyzer) device:

$\begin{array}{ll}\text { Command } & \text { : MCA ACQUIRE OFF } \\ \text { Purpose } & \text { : It stops MCA acquiring } \\ \text { Format } & : \text { mca acquire off } \\ \text { Example } & : \text { mca acquire off }\end{array}$




\begin{tabular}{|c|c|}
\hline Command & : MCA ACQUIRE <mode> \\
\hline Purpose & : It setups MCA to be in PHA mode or MCA \\
\hline Format & : mca acquire $<$ mode $>$ \\
\hline Example & $\begin{array}{l}\text { mca acquire pha } \\
: \text { mca acquire mcs }\end{array}$ \\
\hline Command & : MCA ERASE \\
\hline Purpose & : It erases MCA memory \\
\hline Format & : mca erase <all $>$ \\
\hline Example & $\begin{array}{l}\text { : mca erase (erase the current memory group) } \\
\text { : mca erase all (erase all memory group) }\end{array}$ \\
\hline
\end{tabular}

Command : MCA ROI (?)

Purpose : It sets the MCA's Region Of Interest.

Format $\quad:$ mca roi $<$ mode $>$ [<region>] [all ROI\#]

The option [all] is used only if <mode $>$ is clear or set.

: If [all] is used for <clear>, it will clear all ROIs in all memory groups.

: If [all] is used for $\langle$ sed, it will set the ROI number in all memory groups, and the specified region is an offset from the first channel of each group.

Example $\quad$ : mca roi on (display regions of interest)

: mca roi off (remove ROIs from display)

: mca roi clear (clear all ROIs in current memory group)

: mca roi set 100120 ( mark region $<100200>$ as a ROI)

$\begin{array}{ll}\text { Command } & \text { : MCA SAVE } \\ \text { Purpose } & : \text { It stores MCA's current memory group data in a binary file } \\ \text { Format } & : \text { mca save < filename> [OVERWRITE] [ALL] }\end{array}$

If the option [OVERWRITE] is used, a previously existing file of the same name is overwritten. Otherwise, the existing files will be protected. If the option [all] is used, it will save all memory group data.

Example : mca save databin 


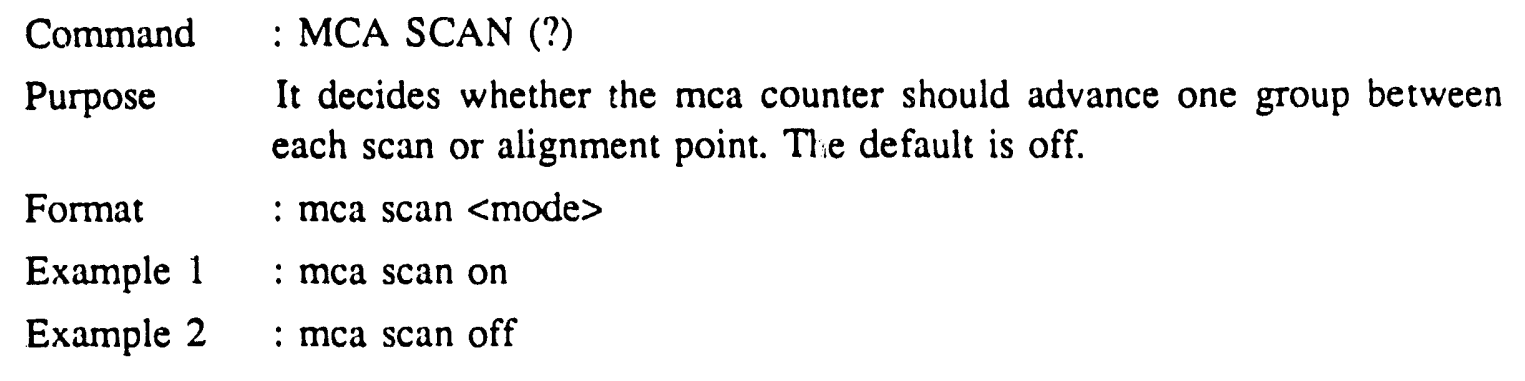
Command : MCA SELECT (?)
Purpose : It selects the memory group for the scan and display.
Format : mca select <group>
Example : mca select HO (It selects group zero (4096 channels) among the two groups.)
: mca select Q1 (It selects group one of the four groups.)
: mca select E2 (It selects group two of the Eight groups.)
: mca select S5 (It selects group five of the Sixteen groups.)
: mca select $T 7$ ( It selects group seven of the thirty-two groups.)
Command : MCA TIME ACQUIRE
Purpose : It starts MCA acquiring data with a real time data graph unless' $-n$ ' is specified.
Format $1 \quad$ : mca time acquire $<$ seconds $>[-n]$
Format 2 : mca time <mode>
Example 1 : mca time acquire 0 (MCA starts acquiring until an OFF command to stop)
: mca time acquire $10-n$ (MCA acquires data for 10 seconds without plot)

Example 2 : mca time live

: mca time real

The following is an example of using an MCA's region of interest as a counter in a scan. The descriptions are included in (). Make sure the MCA roi is included in the counter database file as a counter and enabled for counting (see section 1.3.2 for details).

newfile test ( new data file scan.xxx for all motors' pos. and counts)

mca scan off

mca erase all (erase all of MCA-channels' data)

align th (start scan) 
$-33-$

mca save kate all (save all MCA-groups' spectrum data in new file kate.xxx) 


\section{4 $\mathrm{X}$ window Interclient Communication (?)}

Besides sending commands through keyboards, users can send commands to xexp program through their own programs from other windows by calling function xcom_wrt_str(). The detail procedures are as followings:

1: Users can send any command to the ace program by calling function xcom_wrt_str() passing the command string as the parameter.

Example: $x$ com_wrt_str("mr tt 0.1 ") or xcom_wrt_str("scan.mac").

The above "scan.mac" is a macro file created inside or outside a user's own program.

2: In the user's makefile, they have to call libraries in the following order: - $1 x$ com -IX11 -Insls_s.

3: Before executing, make sure the ACE program is running in the root window. At the end of communication, the screen will echo "done!". 


\section{CHAPTER 2}

\section{How to get around in UNIX ?}

There are too many UNIX commands for our busy users to remember. Besides, many of the commands are user-unfriendly. After tailoring the UNIX commands, we can get around easier. The commands mentioned in this chapter are consist of the standard UNIX commands and the user-defined commands.

\subsection{Floppy disk drive - Bread and Butter}

If you have difficulty in using floppy diskettes drive, just remember how easy life is with 'bread and butter'. Where there is 'bread and butter', there is 'aread and autter'. 'bread, butter or bwrite' read, append, or write data to the $3.5 " 1.44 \mathrm{M}$ bytes double side, high density floppy disk. The commands 'aread, autter or awrite' read, append, or write data to the 5.25" $1.2 \mathrm{M}$ bytes double side, high density Hoppy disk. The wild card usage for pathname applies as usual. As regards to other kinds of floppy disks, please see your system administrator.

In UNIX environment:

aformat $=$ format $/ \mathrm{dev} / \mathrm{rdsk} / \mathrm{fOq} 15 \mathrm{dt}:$ format the disk on a 5.25 " disk drive.

bformat $=$ format $/ \mathrm{dev} / \mathrm{rdsk} / \mathrm{flq} 18 \mathrm{dt}$ : format the disk on a $3.5^{\prime \prime}$ disk drive.

adir $=\operatorname{tar} \operatorname{tvf} /$ dev/fd096: dir files in the floppy disk of the driver

$\operatorname{aread}=\operatorname{tar} \mathrm{xvf} / \mathrm{dev} / \mathrm{fd} 096$ : read out files from the floppy disk.

example:

aread (read out all files from the disk)

aread *.c

aread pathname (read out one file from the disk)

(p.s. It will overwrite the files with the same name in the current directory.)

awrite $=\operatorname{tar} \mathrm{cvf} / \mathrm{dev} / \mathrm{fd} 096$ : write files into the floppy disk.

example:

awrite *.c (wildcard applies, it reads all $C$ files th the disk)

awrite pathname (read out one file from the disk)

(p.s. It will overwrite any file which was originally in the disk)

On some computers with two floppy drives, the command will be aformat, aread, awrite for drive $A$, and bformat, bread, bwrite for drive $B$ instead of aformat, aread, awrite. 
In DOS under UNIX:

Please trpe "vpix" to enter the bridge between DOS and UNIX. In vpix, you can access floppy diskettes drive the same way as DOS (A:).

You can copy binary files directly between DOS and UNIX, but you have to specify a parameter to copy text files between DOS and UNIX.

To copy a DOS-format text file from a: driver to the hard disk, you can do the followings:

copy / $\mathrm{u}$ a:pathname pathname

(where /u means copying a DOS-format file into a UNIX format file) ${ }^{r}$

To copy a UNLX-format text file from the hard disk to a: driver, you can do the followings:

copy /d pathname a:pathname

\subsection{Other commonly used commands}

pwd print the current working director

cd change the current working directory

\subsection{Norton Utilities}

Note: Norton utilities CAN NOT recover all the files deleted accidentally!

It will recover the file which was unique and was deleted with the command "rm filename". If the file was overwritten,it is impossible to recover it.

To recover the file you deleted, type "nue filename". To get a menu-driven interface with help information, speed search, and command history for the Norton Utilities, type "ni".

\section{$2.4 \mathrm{X}$ window commands}

To enter $\mathrm{X}$ window, type 'xinit'.

To create another window, you can do one of the followings: To close a window, you can just logout the window by moving cursor to the window, and then type ${ }^{\wedge} \mathrm{D}$ (CTRL+D).

\subsection{Networking}

To find out your computer system name, please type 'uname $-n$ '. 


\subsubsection{Transfer files to and from a remote UNIX system host}

To transfer file through Ethernet, please type 'ftp -n nodename' (e.x.: ftp - $n$ bnlls 1 ) . The remote nodename must be a UNIX system. Then type 'user' to get the prompt (username), and type in your login name, followed with an ENTER and your password. Now you can use 'cd' to change the remote computer's directory, use 'put pathname' to put a file to the remote computer, use simply type 'bye'.

\subsection{2 login to a remote UNIX host}

To login to a remote UNIX host, please use 'rlogin host -l username'. One example is 'rlogin bnlls 1 -1 feng: From host bnlls 1 , you can login to a VAX/VMS host using 'dlogin hostname'. 

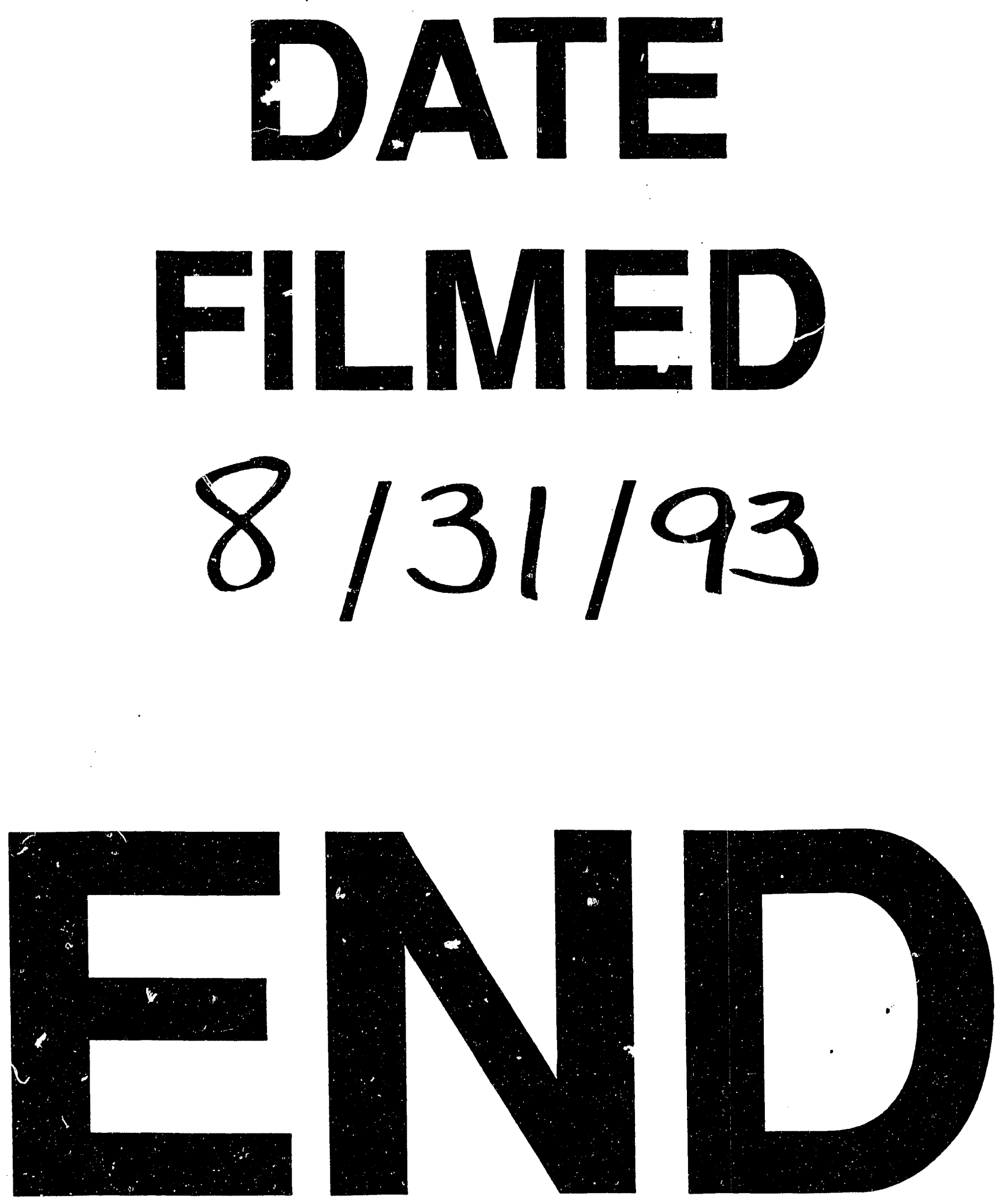
